02

\title{
Исследование твердотельной структурной динамики с помощью сверхбыстрой электронной дифракции и микроскопии
}

\author{
(C) Б.Н. Миронов ${ }^{1}$, С.А. Асеев ${ }^{1}$, С.В. Чекалин ${ }^{1}$, А.А. Ищенко ${ }^{2}$, В.О. Компанец ${ }^{1}$, Е.А. Рябов ${ }^{1}$ \\ ${ }^{1}$ Институт спектроскопии РАН, \\ 108840 Троицк, Москва, Россия \\ ${ }^{2}$ Российский технологический университет - МИРЭА, \\ 119454 Москва, Россия \\ e-mail: mironov@isan.troitsk.ru
}

Поступила в редакцию 25.02.2019 г.

В окончательной редакции 25.02.2019 г.

Принята к публикации 15.03.2019 г.

Продемонстрированы прямые методы отображения возбуждённого фемтосекундным лазером вещества в пространственно-временном континууме, основанные на зондировании образца ультракороткими электронными сгустками, строго синхронизованными с фемтосекундными лазерными импульсами. Представлен ряд экспериментов в области сверхбыстрой электронной микроскопии и дифракции, выполненных в Институте спектроскопии РАН.

Ключевые слова: ультракороткие электронные пучки, фемтосекундные лазерные импульсы, сверхбыстрая микроскопия, дифракция.

DOI: $10.21883 /$ OS.2019.07.47926.44-19

\section{Введение}

Исследование лазерно-индуцированных явлений с помощью пико-фемтосекундных электронных сгустков, синхронизованных с лазерными импульсами, открывает возможность наблюдения когерентной структурной динамики материи с высоким пространственно-временным разрешением в целях контроля далеких от равновесия процессов, а также проверки новых теоретических подходов к описанию вещества в связке триады „структурадинамика-функция“" [1-6]. В отличие от чисто оптических методов возбуждения-зондирования использование ультракоротких фотоэлектронных (либо рентгеновских) импульсов позволяет напрямую детектировать сверхмалые атомно-молекулярные перемещения в исследуемом образце, индуцированные мощным лазерным излучением, и тем самым получить прямую информацию о структурной динамике непосредственно из зависящей от времени дифракционной картины. Важным достоинством метода сверхбыстрой электронной дифракции и микроскопии является возможность использования настольной экспериментальной установки, существенно более доступной, если сравнивать её с синхротронами и лазерами на свободных электронах. Однако для изучения когерентной динамики в твёрдом теле методом просвечивающей электронной микроскопии и дифракции необходимы образцы высокого качества с характерной толщиной $\sim 10-100 \mathrm{~nm}$. Методы, использующие ультракороткие электронные и рентгеновские импульсы для прецизионной диагностики лазерно-возмущённой материи, обладают своими уникальными характеристиками; их подробное сравнение представлено в [7]. Здесь в качестве примера заметим, что сечение упругого рассеяния электронов с энергией $100 \mathrm{keV}$ для алюминия в $\sim 10^{5}$ раз превышает сечение рассеяния электромагнитного излучения на длине волны $1.24 \AA$, а зондирование вещества импульсным электронным пучком оказывается менее разрушительным для образца [8]. Вместе с тем кулоновское расталкивание нерелятивистских электронов (с энергией менее $300 \mathrm{keV}$ ) препятствует формированию плотных электронных импульсов сверхкороткой длительности, что создаёт серьёзные трудности при достижении временного разрешения $<100$ fs [9-11].

Сверхбыстрая электронная дифракции и микроскопия открывают уникальные возможности исследования сверхбыстрых фазовых переходов, состояний вещества, далёких от равновесия, включая экстремальные состояния, прямого наблюдения лазерно-индуцированных фононов в различных структурах, а также атомно-молекулярных перемещений в белках ит.д. на сравнительно компактных экспериментальных установках [1-6]. В Институте спектроскопии РАН развиваются экспериментальные подходы, нацеленные на изучение лазерно-индуцированной структурной динамики в веществе с помощью ультракоротких электронных импульсов, позволяющих проследить состояние материи в пространственно-временном континууме с высокой степенью детализации. На созданном нами фемтосекундном твердотельном электронографе и реконструированном сверхбыстром просвечивающем электронном микроскопе проведен ряд исследований, которые изложены в настоящей работе. 

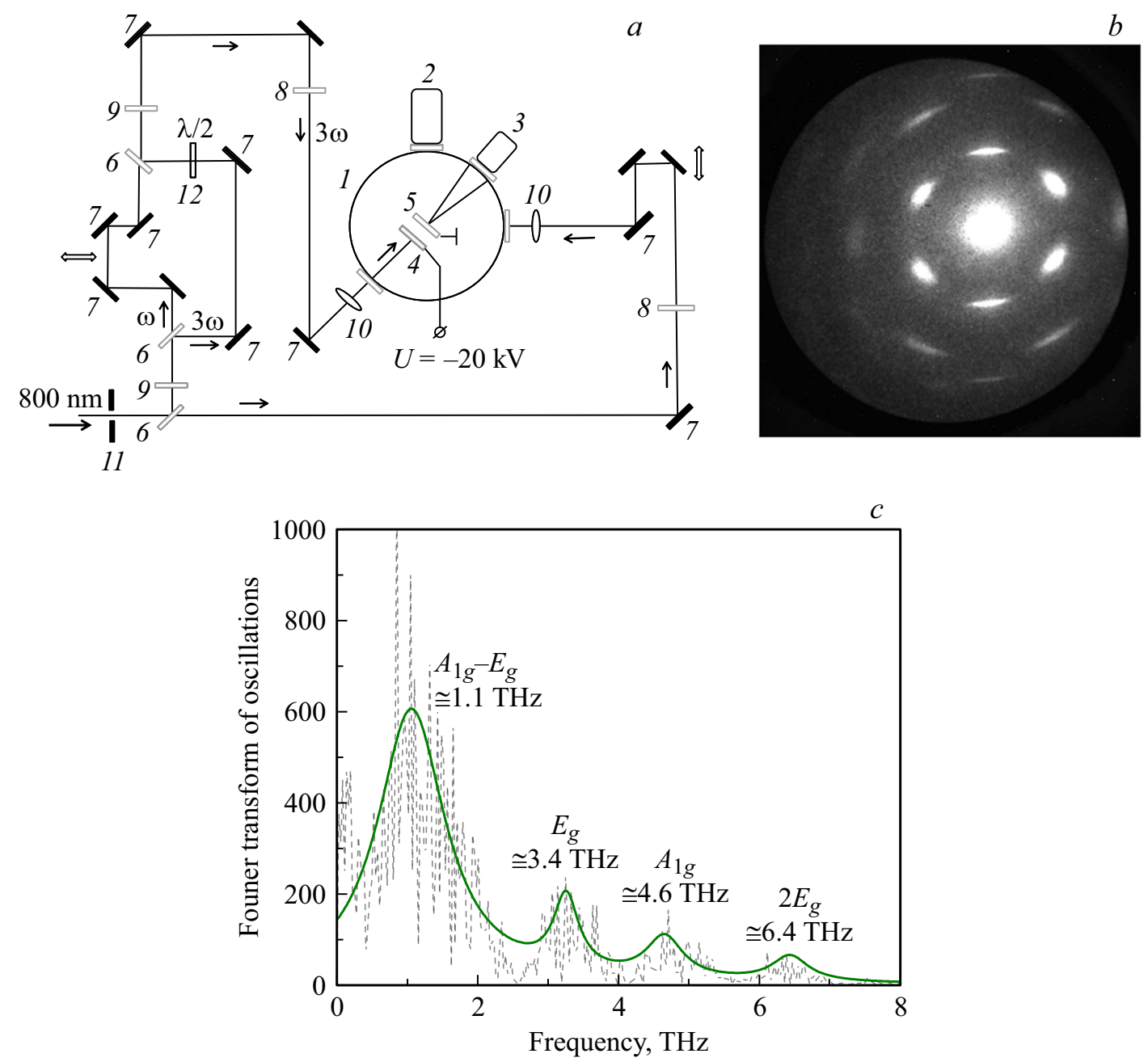

Рис. 1. (а) Принципиальная схема сверхбыстрого твёрдотельного электронографа: 1 - вакуумная камера, 2 - турбомолекулярный насос, 3 - детектор электронов на основе МКП и ПЗС-камеры, 4 - серебряный фотокатод, 5 - анод + мишень, 6 - делители лазерного излучения, 7 - зеркала, 8 - ослабитель излучения, 9 - преобразователи лазерного излучения во вторую и третью гармоники, 10 - линзы, 11 - диафрагма, 12 - вращатель плоскости поляризации лазерного излучения. (b) Дифрактограмма тонкой плёнки сурьмы, полученная с помощью фотоэлектронных импульсов с энергией $20 \mathrm{keV}$. (c) Фурьеспектр лазерно-индуцированных осцилляций дифракционного сигнала в пленке $\mathrm{Sb}$ (пунктирная линия - эксперимент, сплошная линия - аппроксимация экспериментальных данных функциями Лоренца).

\section{Исследования лазерно-индуцированных процессов в сурьме и кобальтите лантана на фемтосекундном электронографе}

Принципиальная схема настольного фемтосекундного электронографа $[12,13]$ показана на рис. $1, a$. В отличие от фемтосекундной лазерной спектроскопии здесь использован несколько иной подход: в методе сверхбыстрой электронной дифракции для зондирования лазерноиндуцированных процессов в веществе применены электронные импульсы, формируемые при облучении фотокатода фемтосекундным лазерным излучением и посылаемые на образец в момент времени, определяемый линией задержки.
В собранном приборе источником фотоэлектронов является слой золота (или серебра) 30 nm на кварцевой пластине, облучаемый третьей гармоникой титансапфирового лазера с длиной волны $266 \mathrm{~nm}$, а для возбуждения образца использована основная гармоника этого же лазера с длиной волны $800 \mathrm{~nm}$ и длительностью импульса около $50 \mathrm{fs}$. Использование единого задающего фемтосекундного лазера для обоих каналов обеспечивает прецизионную синхронизацию оптических и фотоэлектронных импульсов. Фотоэлектронный пучок с кинетической энергией $20 \mathrm{keV}$ и диаметром $0.1 \mathrm{~mm}$ на образце формируется ускоряющим высоким электростатическим полем и специально подобранной линзой на основе постоянного магнита. Это позволяет достичь сверхмалого времяпролетного промежутка $12 \mathrm{~mm}$ от катода до образца, минимизировать расплывание элек- 
тронного облака и тем самым обеспечить высокое временное разрешение $\sim 300 \mathrm{fs}$ при $\sim 10^{3}$ электронов в импульсе. Для детектирования электронов использована позиционно-чувствительная схема на базе пары микроканальных пластин (МКП) и люминофорного экрана, информация с которого считывалась CCD-камерой и передавалась в компьютер.

Для экспериментов в качестве одного из образцов была выбрана сурьма, относящаяся к полуметаллам. Такие материалы характеризуются довольно высокой эффективностью генерации когерентных оптических решёточных колебаний при импульсном лазерном воздействии на образец, что впервые наблюдалось в чисто лазерных экспериментах. Для исследования этих процессов методом сверхбыстрой электронной дифракции на просвет пленка сурьмы толщиной $\sim 30 \mathrm{~nm}$ наносилась термическим способом на углеродную подложку, применяемую в просвечивающей электронной микроскопии.

Дифракционная картина от тонкой пленки сурьмы, полученная с помощью импульсного фотоэлектронного пучка в отсутствие оптического возбуждения, характеризуется наличием ярких рефлексов, что однозначно свидетельствует о кристаллической природе этого образца (рис. 1,b). При лазерном возбуждении образца (плотностью энергии в лазерном импульсе около $1.5 \mathrm{~mJ} / \mathrm{cm}^{2}$, частота повторения $1 \mathrm{kHz}$ ) значения сигнала в разных областях дифрактограммы нормировались на величину сигнала в отсутствие лазерного возбуждения и анализировались затем в зависимости от времени задержки между возбуждающим лазерным и зондирующим электронным импульсами. Полученная с шагом $60 \mathrm{fs}$ и накоплением, равным $1 \mathrm{~s}$, временная зависимость характеризовалась явно выраженной осциллирующей компонентой с периодом на пикосекундной шкале. Из фурье-анализа экспериментальных данных следует, что такая модуляция вызвана возбуждением ансамбля мод с частотами $1.1,3.4,4.6$ и $6.4 \mathrm{THz}$, три из которых наблюдались ранее в чисто оптических экспериментах (рис. 1,c) [14,15]. Следуя [14,15], частоты 4.5 и $3.5 \mathrm{THz}$ принадлежат полносимметричным $A_{1 g}$ и дважды вырожденным $E_{g}$ оптическим фононам $\mathrm{Sb}$, а $1.1 \mathrm{THz}$ можно приписать разностным оптическим колебаниям $A_{1 g}-E_{g}$. Для сурьмы частота $6.4 \mathrm{THz}$, зарегистрированная в данном эксперименте, ранее не наблюдалась, и ее можно приписать второй гармонике фононной моды $E_{g}$. В этом эксперименте впервые реализовано прямое наблюдение генерации когерентных оптических фононов в сурьме методом сверхбыстрой электронной дифракции [12,13]. Теоретические и экспериментальные исследования сфазированных оптических колебаний решётки в этом полуметалле мощными фемтосекундными лазерными импульсами были продолжены немецкими коллегами в работах $[16,17]$.

В качестве еще одного образца был выбран кобальтит лантана $\left(\mathrm{LaCoO}_{3}\right)$. Это соединение, иногда обозначаемое как LCO, достаточно давно представляет интерес для широкого круга экспериментаторов и теоретиков в области физики твёрдого тела и материаловедения [1824]. Установлено, что данный материал обладает аномальными магнитными, электронными и транспортными свойствами; однако остаются не до конца выясненными природа основного состояния $\mathrm{LaCoO}_{3}$ и изменения его свойств, происходящие по мере роста температуры образца. Специфика „аномальных“ характеристик LCO вызвана совокупностью различных взаимодействий в рассматриваемой системе, обусловленных зарядовыми, спиновыми и орбитальными степенями свободы. Так, для иона кобальта в этом перовските возможны одновременно несколько типов спинового состояния и несколько типов зарядового состояния. Экспериментальные данные указывают на две важные модификации электронной структуры $\mathrm{LaCoO}_{3}$, происходящие по мере роста температуры системы: диамагнитный-парамагнитный спиновый переход около $100 \mathrm{~K}$ и фазовый переход полупроводник-металл между 350 и 650 К. Несмотря на большое количество работ в области физики кобальтита лантана, единое понимание электронных свойств и изменений магнитной конфигурации этой системы продолжает разрабатываться $[25,26]$.

Наночастицы кобальтита лантана со средним размером 65-70 nm были синтезированы на химическом факультете МГУ имени М.В. Ломоносова с использованием „золь-гель“ технологии. Отжиг при температуре $1000 \mathrm{~K}$ служил для повышения степени кристалличности материала. Использование изопропилового спирта и ультразвука позволило достичь оптимального распределения частиц LCO на поверхности углеродной пленки после высыхания раствора. Электронограмма от наночастиц кобальтита лантана представлена на рис. 2, a. Заметим, что картина электронной дифракции получена в условиях, когда кристаллографические плоскости в образце ориентированы случайным образом.

В эксперименте регистрировалось изменение во времени дифракционной картины, вызванной возбуждением LCO мощными лазерными импульсами с плотностью энергии $\sim 0.5 \mathrm{~mJ} / \mathrm{cm}^{2}$ (рис. $2, b$ ); остальные условия эксперимента были идентичны описанным выше при исследовании тонких плёнок сурьмы. Отметим характерный масштаб сигнала $t^{*} \approx 11$ ps на рис. $2, b$. Принимая во внимание, что скорость звука в этом материале $v_{s}=5900 \mathrm{~m} / \mathrm{s}$ [20], получим оценку на характерный пространственный масштаб $l=t^{*} v_{s} \approx 65 \mathrm{~nm}$, что удовлетворительно совпадает с размером наночастиц LCO, измеренным независимым способом. Фурье-анализ экспериментальных данных обнаружил, что наблюдаемая модуляция сигнала обусловлена присутствием моды с частотой осцилляции $\sim 4 \mathrm{THz}$. Согласно исследованиям в области рамановской спектроскопии LCO [27], это вероятно вызвано колебаниями лантана в гексагональной (001) плоскости. Заметим, что за возбуждение когерентных решёточных осцилляций ответственен фемтосекундный лазерный импульс накачки, спектр которого в наших условиях позволяет создавать когерентные фононы с частотами до $\sim 10 \mathrm{THz}$ и выше. Но, в отличие от 

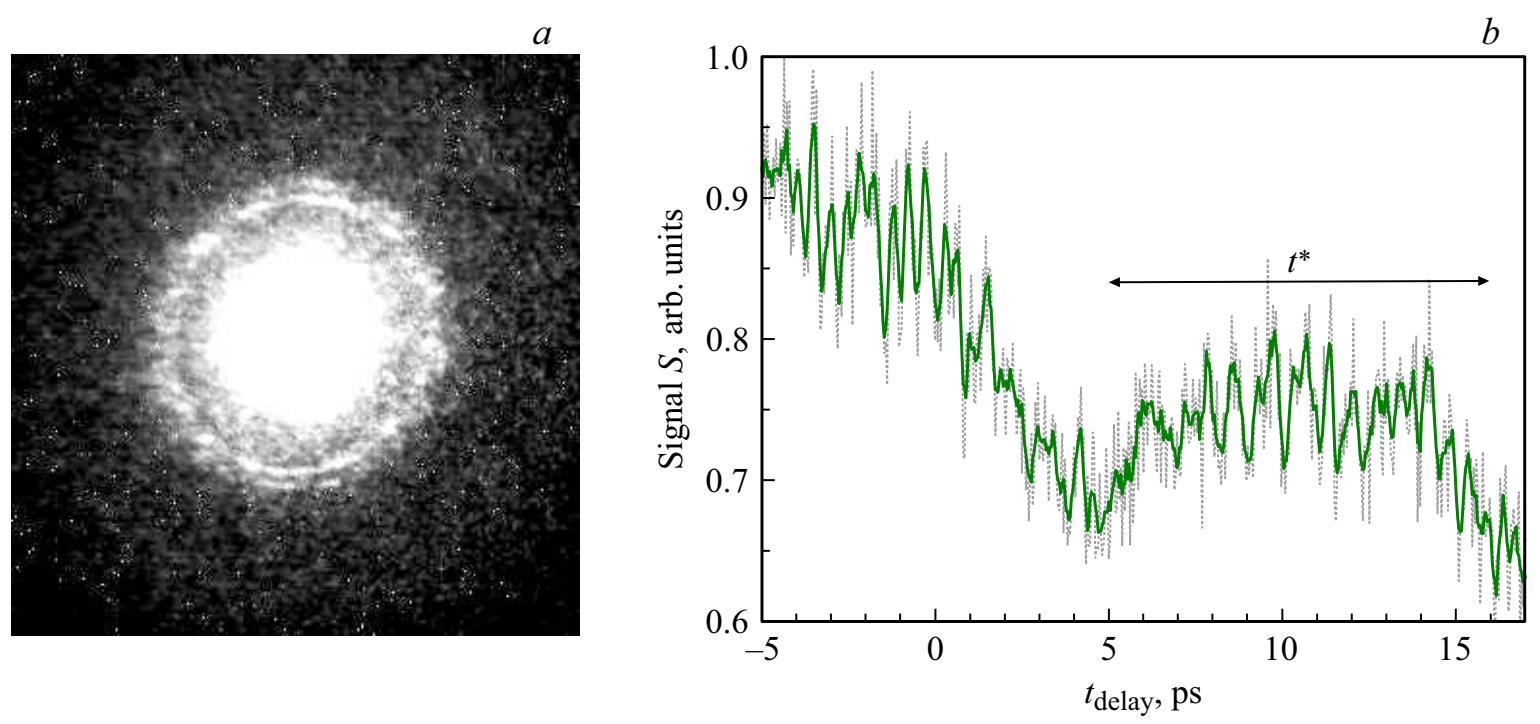

Pис. 2. (a) Картина электронной дифракции от наночастиц LCO, полученная с помощью фотоэлектронных импульсов с энергией $20 \mathrm{keV}$. (b) Зависимость интенсивности электронной дифракции в LCO от задержки между возбуждающим лазерным и зондирующим фотоэлектронным импульсом (пунктирная линия - эксперимент, сплошная линия - усреднение экспериментальных данных по 5 соседним значениям).

чисто оптических методов возбуждения-зондирования, для их разрешения методом сверхбыстрой электронной дифракции необходимо использовать более короткие электронные сгустки (по сравнению с использованными в данном эксперименте).

В экспериментальном аспекте основные трудности работы с LCO обусловлены сравнительно большим размером облучаемых наночастиц: при кинетической энергии $20 \mathrm{keV}$ электронный пучок испытывает сильное поглощение при зондировании микрочастиц диаметром $\sim 70 \mathrm{~nm}$. Поэтому для дальнейшей работы предполагается использовать образцы LCO, характеризующиеся малым размером $\sim 10-20 \mathrm{~nm}$.

\section{Реконструкция сверхбыстрого просвечивающего электронного микроскопа}

Исследование вещества методом электронной дифракции на этапе извлечения информации о структуре образца из наблюдаемой дифрактограммы требует решения обратной задачи. Электронная микроскопия позволяет определять структурные характеристики образца напрямую. Для реконструкции промышленного просвечивающего электронного микроскопа Hitachi H-300 (рис. 3, a), имеющего пространственное разрешение $\sim 5 \AA$ в режиме микроскопии с помощью непрерывного электронного пучка $75 \mathrm{keV}$, необходимо было осуществить замену термического катода на фотокатод, ввести лазерные пучки внутрь колонны в область фотокатода и образца, а также обеспечить контроль пространственно-временного совмещения электронных и оптических импульсов непосредственно в области тонкопленочного образца. Эти задачи были успешно реализованы, а также была произведена замена фотографической регистрации электронов на 2D-детектор на базе люминофора и CCD-камеры [28]. На рис. $3, b$ представлены результаты, полученные в фотоэмиссионном и термоэмиссионном режимах работы. Термоэмиссионный режим является стандартным для Hitachi H-300 и служит удобным репером для оценки функционирования данной установки. В фотоэмиссионной моде для облучения серебряного фотокатода и генерации импульсного фотоэлектронного пучка использовалась вторая гармоника фемтосекундного лазера „Маі-Таі“, работающего с высокой частотой следования импульсов $80 \mathrm{MHz}$. Сходство экспериментальных данных, показанных на рис. $3, b$, является подтверждением работоспособности реконструированного прибора.

Для нахождения „нулевой“ точки использован подход, основанный на трансформации траектории фотоэлектронного пучка $75 \mathrm{keV}$ после его пролёта сквозь достаточно плотное облако медленных электронов вблизи облучённого лазером медного прутка диаметром $0.5 \mathrm{~mm}$. Медный образец закреплён на пересечении пары ортогональных металлических пластин с прорезями шириной $0.1 \mathrm{~mm}$, что облегчало пространственное сведение оптических и электронных пучков. В эксперименте излучение фемтосекундного волоконного лазера „ANTAUS“ на длине волны $1050 \mathrm{~nm}$ преобразовывалось во вторую гармонику для облучения образца (плотность энергии $\sim 5 \mathrm{~mJ} / \mathrm{cm}^{2}$ ) и четвертую для активации фотокатода. Изменяя задержку между этими оптическими каналами, можно было проследить динамику взаимодействия быстрого электронного пучка с плотным ансамблем 

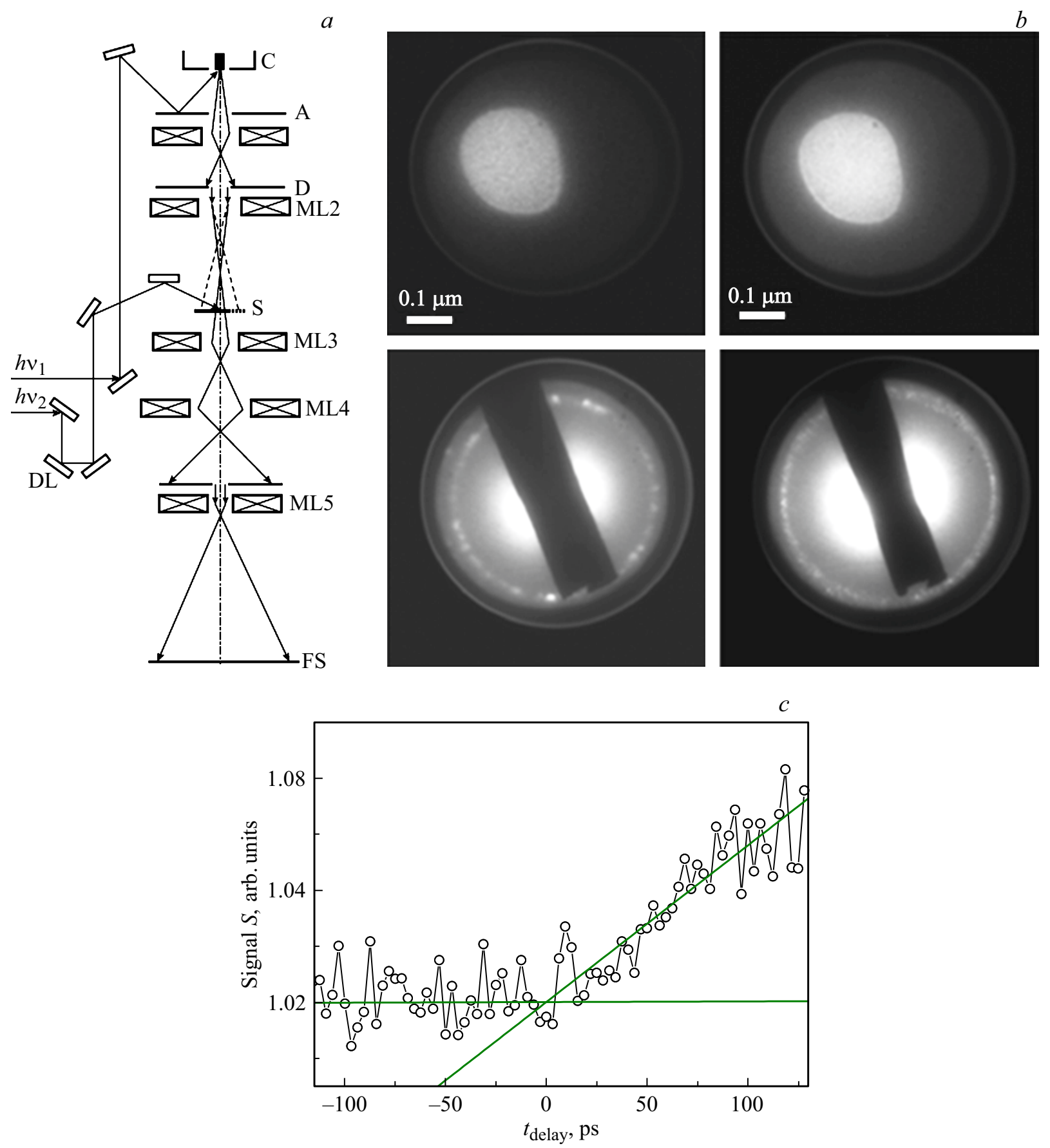

Рис. 3. (a) Принципиальная схема пикосекундного просвечивающего электронного микроскопа: C, А - катод и анод узла эмиссии и ускорения электронов соответственно, ML1, ML2 - магнитные линзы конденсора осветительной системы, D - узел со сменными диафрагмами, S - образец, ML3 - магнитная линза объектива, ML4 - промежуточная линза, ML5 - проектирующая линза, FS - флуоресцентный экран, DL - оптическая линия задержки. (b) Изображения микроотверстия в углеродной плёнке и дифракционные картины от алюминиевых наночастиц, полученные в фотоэмиссионном (слева) и стандартном термоэмиссионном

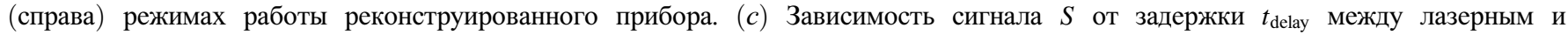
фотоэлектронным импульсом: аппроксимация экспериментальных данных дана двумя сплошными линиями в соответствии с методом [29].

медленных электронов [28]. В рамках выполненного эксперимента нулевая точка отсчёта определяется пересечением двух прямых, одна из которых соответствует плато, а другая - переднему фронту изменения сигнала (рис. 3,c) [29]. Найденное таким образом положение нулевой точки оказалось размытым на величину около $10 \mathrm{ps,}$ что близко к численной оценке длительности электронного импульса, равной 7.5 ps [28]. 
Таким образом, при реконструкции промышленного просвечивающего электронного микроскопа в режиме использования импульсного фотоэлектронного пучка достигнуто пикосекундное временное разрешение. В сочетании с атомным пространственным разрешением это открывает возможности прямого наблюдения лазерноиндуцированной структурной динамики в белках, ранее проводившихся с субнаносекундным временным разрешением на синхротроне в Гренобле [30].

\section{Заключение}

Развитие методов сверхбыстрой электронной дифракции и микроскопии представляет огромный интерес для детального исследования далёких от равновесия быстропротекающих процессов и структурно-фазовых лазерно-индуцированных переходов в физике, химии и биологии на их естественных пространственно-временных масштабах. Для этого в Институте спектроскопии РАН подготовлены две экспериментальные установки, которые в настоящее время являются единственными в нашей стране. На фемтосекундном электронографе впервые выполнены исследования когерентных оптических фононов в сурьме методом сверхбыстрой электронной дифракции и проведена первая серия измерений лазерно-индуцированных процессов в кобальтите лантана. На базе промышленного прибора Hitachi H-300 создан сверхбыстрый просвечивающий электронный микроскоп, который позволяет проводить исследования структурной динамики в сложных органических соединениях для получения информации, выходящей за рамки возможностей чисто оптических измерений.

\section{Благодарности}

Эксперименты выполнены на УНУ „Многоцелевой фемтосекундный лазерно-диагностический спектрометрический комплекс“ Института спектроскопии Российской Академии Наук.

\section{Конфликт интересов}

Авторы заявляют, что у них нет конфликта интересов.

\section{Список литературы}

[1] Zewail A.H., Thomas J.M. 4D Electron Microscopy. Imaging in Space and Time. London: Imperial College Press, 2010. $360 \mathrm{p}$.

[2] Ищенко А.А., Гиричев Г.В., Тарасов Ю.И. Дифракция электронов: Структура и динамика свободных молекул и конденсированного состояния вещества. М.: Физматлит, 2013. $614 \mathrm{c}$.

[3] Ischenko A.A., Aseyev S.A. Time Resolved Electron Diffraction: for Chemistry, Biology and Materials Science. USA, San Diego: Elsevier, 2014. 274 p.
[4] Ищенко А.А., Асеев С.А., Баграташвили В.Н., Панченко В.Я., Рябов Е.А. // УФН. 2014. Т. 184. С. 681. doi 10.3367/UFNr.0184.201407a.0681

[5] Miller R.J.D. // Ann. Rev. Phys. Chem. 2014. V. 65. P. 583. doi 10.1146/annurev-physchem-040412-110117

[6] Ischenko A.A., Weber P.M., Miller R.J.D. // Chem. Rev. 2017. V. 117. P. 11066. doi 10.1021/acs.chemrev.6b00770

[7] Chergui M., Zewail A.H. // Chemphyschem. 2009. V. 10. P. 28. doi $10.1002 /$ cphc.200800667

[8] Spence J.C.H. // Struct. Dynamics. 2017. V. 4. P. 044027. doi 10.1063/1.4984606

[9] van Oudheusden T., Pasmans P.L.E.M., van der Geer S.B., de Loos M.J., van der Wiel M.J., Luiten O.J. // Phys. Rev. Lett. 2010. V. 105. P. 264801. doi 10.1103/PhysRevLett.105.264801

[10] van Oudheusden T., Pasmans P.L.E.M., van der Geer S.B., de Loos M.J., van der Wiel M.J., Luiten O.J. // Ultrafast Phenomena XVI. / Ed. by Corkum P., Silvestri S., Nelson K.A., Riedle E., Schoenlein R.W. Berlin, Heidelberg: Springer-Verlag, 2009. P. 938.

[11] Kochikov I.V., Miller R.J.D., Ischenko A.A. // ЖЭТФ. 2019.

[12] Миронов Б.Н., Компанеи В.О., Асеев С.А., Ищенко А.А., Мисочко О.В., Чекалин С.В., Рябов Е.A. // Письма в ЖЭТФ. 2016. Т. 103. С. 597. doi 10.7868/S0370274X16080075

[13] Миронов Б.Н., Компанеи, В.О., Асеев С.А., Ищенко А.А., Кочиков И.В., Мисочко О.В., Чекалин С.В., Рябов Е.А. // ЖЭТФ. 2017. Т. 151. С. 494. doi $10.7868 / \mathrm{S} 0044451017030051$

[14] Ishioka K., Kitajima M., Misochko O.V. // J. Appl. Phys. 2008. V. 103. P. 123505 . doi $10.1063 / 1.2940130$

[15] Chekalin S.V., Melnikov A.A., Misochko O.V. // Laser Phys. 2014. V. 24. P. 094004. doi 10.1088/1054-660X/24/9/094004

[16] Waldecker Lutz, Vasileiadis Thomas, Bertoni Roman, Ernstorfer Ralph, Zier Tobias, Valencia Felipe H., Garcia Martin E., Zijlstra Eeuwe S. // Phys. Rev. B. 2017. V. 95. P. 054302. doi 10.1103/PhysRevB.95.054302

[17] Bauerhenne B., Zijlstra E.S., Garcia M.E. // Appl. Phys. A. 2017. V. 123. P. 608. doi 10.1007/s00339-017-1216-7

[18] Royer Sébastien, Duprez Daniel, Can Fabien, Courtois Xavier, Batiot-Dupeyrat Catherine, Laassiri Said, Alamdari Houshang // Chem. Rev. 2014. V. 114. P. 10292. doi 10.1021/cr500032a

[19] Imada M., Fujimori A., Tokura Y. // Rev. Mod. Phys. 1998. V. 70. P. 1039. doi 10.1103/RevModPhys. 70.1039

[20] Izquierdo M., Karolak M., Trabant C., Holldack K., Föhlisch A., Kummer K., Prabhakaran D., Boothroyd A.T., Spiwek M., Belozerov A., Poteryaev A., Lichtenstein A., Molodtsov S.L. // Phys. Rev. B. 2014. V. 90. P. 235128. doi 10.1103/PhysRevB.90.235128

[21] Bielecki J., Rata A.D., Börjesson L. // Phys. Rev. B. 2014. V. 89. P. 035129. doi 10.1103/PhysRevB.89.035129

[22] Radaelli P.G., Cheong S.-W. // Phys. Rev. B. 2002. V. 66. P. 094408. doi 10.1103/PhysRevB.66.094408

[23] Haverkort M.W., Hu Z., Cezar J.C., Burnus T., Hartmann H., Reuther M., Zobel C., Lorenz T., Tanaka A., Brookes N.B., Hsieh H.H., Lin H.-J., Chen C.T., Tjeng L.H. // Phys. Rev. Lett. 2006. V. 97. P. 176405. doi 10.1103/PhysRevLett.97.176405

[24] Klie R.F., Yuan T., Tanase M., Yang G., Ramasse Q. // Appl. Phys. Lett. 2010. V. 96. P. 082510. doi 10.1063/1.3336010

[25] Chakrabarti B., Birol T., Haule Kr. // Phys. Rev. Materials. 2017. V. 1. P. 064403. doi 10.1103/PhysRevMaterials.1.06440 
[26] Chakrabarti B., Birol T., Haule Kr. // arXiv. Role of Entropy and Structural Parameters in the Spin-state Transition of LaCoO3. Dated: October 15, 2018.

[27] Gnezdilov V., Choi K.-Y., Pashkevich Yu., Lemmens P., Shiryaev S., Bychkov G., Barilo S., Fomin V., Yeremenko A.V. // Low Temp. Phys. 2006. V. 32. P. 162. doi 10.1063/1.2171521

[28] Андреев С.В., Асеев С.А., Баграташвили В.Н., Воробьев Н.С., Ищенко А.А., Компанеи В.О., Малиновский А.Л., Миронов Б.Н., Тимофеев А.А., Чекалин С.В., Шашков Е.В., Рябов Е.А. // Квант. электрон. 2017. Т. 47. C. 116. doi 10.1070/QEL16276

[29] Badali D.S., Gengler R.Y.N., Miller R.J.D. // Struct. Dyn. 2016. V. 3. P. 034302 . doi $10.1063 / 1.4949538$

[30] Schotte Friedrich, Lim Manho, Jackson Timothy A., Smirnov Aleksandr V., Soman Jayashree, Olson John S., Phillips Jr. George N., Wulff Michael, Anfinrud Philip A. // Science. 2003. V. 300. P. 1944. doi 10.1126/science.1078797 